\title{
The soft X-ray characteristics of solar flares, both with and without associated CMEs
}

\author{
H. R. M. Kay, L. K. Harra, S. A. Matthews, J. L. Culhane, and L. M. Green \\ Mullard Space Science Laboratory, University College London, Holmbury St. Mary, Dorking, Surrey, RH5 6NT, UK
}

Received 9 December 2002 / Accepted 9 January 2003

\begin{abstract}
The complex relationship between solar flares and coronal mass ejections is investigated using a comparison of flare parameters for a total of 69 ejective and non-ejective flares. In the case of solar flares which do not show mass ejection there seems to be a clear relationship between the peak intensity and duration, with higher intensity events being of longer duration. Systematic differences in the relationship between peak temperature and intensity for the two types of event are also evident, with flares associated with CMEs tending to have lower peak temperatures than non-ejective events of the same intensity. Whilst there appears to be a clear relationship between the length of rise and decay phase in a flare, there are no systematic differences in these parameters for ejective and non-ejective flares. A total of eleven "EIT waves" were found, all of which were associated with CMEs. There is no apparent correlation between the occurrence of an EIT wave and the peak temperature, intensity or rise time of the associated flare.
\end{abstract}

Key words. Sun: activity - Sun: flares - Sun: coronal mass ejections (CMEs)

\section{Introduction}

The complex relationship between solar flares and coronal mass ejections (CMEs) has been studied intensively, but as yet has not been fully explained. Although it has long been known that the two phenomena often occur in conjunction, the relationship is not one-to-one and the exact nature of the flare and CME triggers is not known.

Whilst statistical studies indicate that higher intensity events are more likely to be accompanied by a CME (Harrison 1995), this is not always the case. For example Green et al. (2002) present analysis of an X1.2 class flare for which there was no associated CME.

Many flares with associated CMEs have been found to be long duration events, indicating a possible relation between flare duration and mass ejection. As a result Sheeley (1983) looked at this relation for GOES classes $\mathrm{C}$ to $\mathrm{X}$ events with durations greater than $30 \mathrm{~min}$. While they found that long duration events ( $>5$ hours) were all associated with CMEs, so were some short duration events. Subsequent work by Harrison (1995) again confirmed that CMEs could be associated with flares of any duration, but that long duration events were more likely to have associated mass ejections, as were higher intensity flares.

The temporal relationship between the two types of event is also not clear. Zhang (2001) investigated this for four events

Send offprint requests to: $\mathrm{H}$. R. M. Kay,

e-mail: hrm@mssl.ucl.ac.uk on the solar limb and concluded that the initial phase of the CME occurred before the onset of the associated flare in three of the four cases. However, observations of transequatorial interconnecting loops by Khan \& Hudson (2000), suggest that the occurrence of a flare was the driver of both the CMEs and the disappearance of the interconnecting loops.

There have also been a number of statistical investigations into the properties of soft X-ray flares in general, such as temperature, emission measure (EM) and duration (Harrison 1995 and references therein). The peak emission measure and the colour temperature $(T)$, as determined from observations by the Geostationary Operational Environmental Satellite (GOES), have both been found to be correlated with the peak intensity (Feldman et al. 1995a, 1996) and in addition peak emission measures are predicted to vary as $E M \sim T^{7}$ (Aschwanden 1999). Veronig et al. (2002) have used GOES data from 1976 to 2000 to examine the relationship between flare duration, SXR peak intensity and fluence for almost 50000 events. They again find that duration increases with peak intensity. However they do not attempt to differentiate between flares with and without associated CMEs.

The launch of the $\mathrm{SOHO}$ satellite, provided observations with the Extreme-ultraviolet Imaging Telescope (EIT: Delaboudinière et al. 1995) which allows us to look for the signatures of mass ejection lower in the corona, such as coronal dimming (Hudson \& Webb 1997; Sterling \& Hudson 1997). By coupling these data with white light data from the Large 
Angle and Spectrometric Coronograph (LASCO: Brueckner et al. 1995) also on $\mathrm{SOHO}$, which covers 1.1 to 32 solar radii, we are now able to make much more confident associations between individual CMEs and flares.

Here we examine a sample of solar flares, including events both with and without associated CMEs, of GOES Class B to $\mathrm{X}$, and with durations as low as $3 \mathrm{~min}$. We also consider the peak temperature and emission measure of the events, and the relative lengths of the rise and decay phase, so that we may look for the effects of mass ejection on the heating, cooling and energy release mechanisms involved in a flare. We also consider the occurrence of associated EIT waves, in relation to the soft X-ray properties of the events.

\section{Flare parameters}

The solar flares chosen for this study consisted of very simple events, identified from their soft X-ray profile. Complex events with multiple peaks, or those which were truncated by the occurrence of another flare, were excluded. Long duration flares with minor events occurring during the decay phase were not excluded, though it is noted that this may artificially increase the length of the decay phase for some of these events. No effort was made to obtain either a temporally or spatially complete sample and events were initially selected on the basis that we could unambiguously determine whether or not there was an associated CME.

Solar flare parameters were measured using data from the X-Ray Sensor (XRS) on the GOES satellite, which monitors the Sun continuously in two broadband soft X-ray channels covering $0.5-4$ and $1-8 \AA$. The peak intensity and duration of each flare were measured using the $1-8 \AA$ channel. Duration was defined as the time between the point where the gradient of the X-ray flux turned positive, to the point where it either returned to the pre-flare value or where it leveled off, whichever occurred earlier (Fig. 1).

The peak colour temperature and emission measure were determined from the ratio of fluxes in the two GOES channels (with background subtracted), using the standard calibration of Garcia (1994) within Solarsoft. Here the colour temperature is defined as the temperature of an isothermal plasma which would produce the observed ratio of fluxes.

\section{Detection of a CME}

Initially the occurrence of a CME was determined using data from LASCO, which allows the solar corona to be imaged between 1.1 and $32 R_{\odot}$. The presence of a CME, and the associated enhancement in density, is evident in the Thomsonscattered photospheric emission in the LASCO images. As the early stages of a CME are not easily investigated using coronagraph data, initial associations with flares were assigned on the basis of the timing and position angle of the CME.

Where possible EIT data were also used to confirm the presence or lack of a CME, using images taken in the $195 \AA$ bandpass, which is dominated by emission lines of Fe XII with a peak emission near 1.5 MK at coronal densities. In EIT images the occurrence of a mass ejection is characterised by a transient

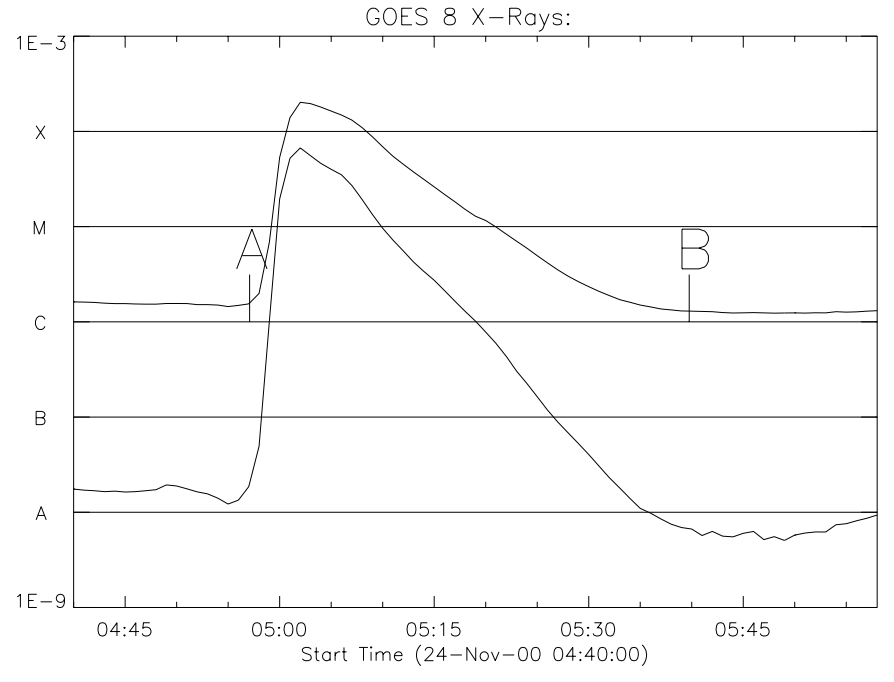

Fig. 1. An example of the SXR profile from GOES for one of the $\mathrm{X}$-class flares, showing the GOES flare classification system. The start and end times of the flares are indicated by the points $\mathrm{A}$ and $\mathrm{B}$ respectively.

decrease in emission, thought to be due to density depletion and evident as a large scale dimming in EIT difference images (Zarro et al. 1999). These regions of dimming have been confirmed as out-flowing material by Harra \& Sterling (2001) who studied spectroscopic observations of one such region using the Coronal Diagnostic Spectrometer (CDS: Harrison et al. 1995) on $\mathrm{SOHO}$. These observations showed Doppler signatures of $\sim 30 \mathrm{~km} \mathrm{~s}^{-1}$ in coronal (Fe XVI and Mg IX) emission lines and $\sim 100 \mathrm{~km} \mathrm{~s}^{-1}$ in transition region (Ov and $\mathrm{He} \mathrm{I}$ ) emission lines.

For cases where there were insufficient EIT data available, only events where the CME could unambiguously be associated to the flare, i.e. no other events occurred which could be related to the CME, were included. In the case of events where no CME was evident and there were insufficient EIT images during the event to look for dimming, EIT images before and after the event were examined for evidence of field lines opening and hence mass ejection. Only events where there appeared to have been no mass ejected were then included in the analysis.

\section{Results and analysis}

\subsection{Soft $X$-ray properties}

The soft X-ray properties of the sample are summarised in Tables 1-3. Figure 2 shows the peak intensity and duration for each of the flares considered. As can be seen there is a general correlation between the duration and the peak intensity for all the events with high intensity events being generally of longer duration. This is consistent with the work of Veronig et al. (2002), although they have used a different method for determining the end point of the flare, based on the point at which the flux returns to half of the peak value. There is no clear dividing line, however, separating the ejective and nonejective solar events. As with the Sheeley et al. work, while events lasting longer than $300 \mathrm{~min}$ do have associated CMEs, 


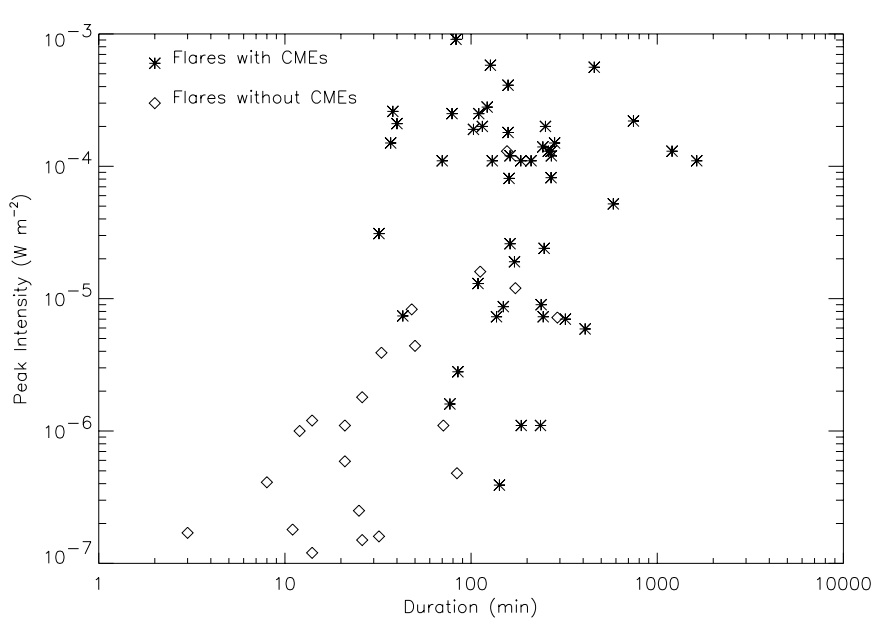

Fig. 2. Peak intensity as a function of duration, measured from the flux in the 1-8 $\AA$ channel of the XRS on GOES.

some events as short as $32 \mathrm{~min}$ also show associated mass ejection. However, the sample of flares with CMEs tend to be, on average, of longer duration, and this trend is seen within each GOES class (Table 2). For Table 1, where the trend seems more pronounced, it is worth noting that the sample is biased towards higher intensity events, due to the inclusion of a large number of X-class flares, some of which are of extremely long duration (>1000 min) which may artificially skew the mean duration.

This correlation between intensity and duration becomes more evident when we consider only those events which do not show mass ejection. The CME associated events appear more randomly scattered with no evidence of any correlation. It appears that the occurrence of a coronal mass ejection, with the often associated large scale restructuring of the coronal magnetic field, intrinsically affects the energy release of the associated flare. In the case of some of the short duration X class flares in Fig. 2, it appears as if the effect of the mass ejection may have caused the release of energy in the flare to occur over shorter timescales, resulting in a shorter flare duration. Similar patterns are seen when considering the peak temperature, which is to be expected given the relationship between peak temperature and intensity observed by Feldman et al. (1995a).

When we consider the peak temperature and peak emission measure of the events (Fig. 3) we see the same correlation as that observed by Feldman et al. (1995a,b) in the case of both solar and stellar flares, with a high peak temperature corresponding to a high peak emission measure. However, there also appear to be systematic differences in this relation (Fig. 3). For a given peak temperature, a higher emission measure is evident for the events with CMEs. Further systematic differences are also clearly seen when we consider the peak temperature as a function of peak intensity, again the two types of event appear separated (Fig. 4), which is supported by the average peak temperatures shown in Tables 2 and 3. However, there is a limited range of temperature and emission measure where there are sufficient numbers of events of both types for a reasonable comparison to be made.

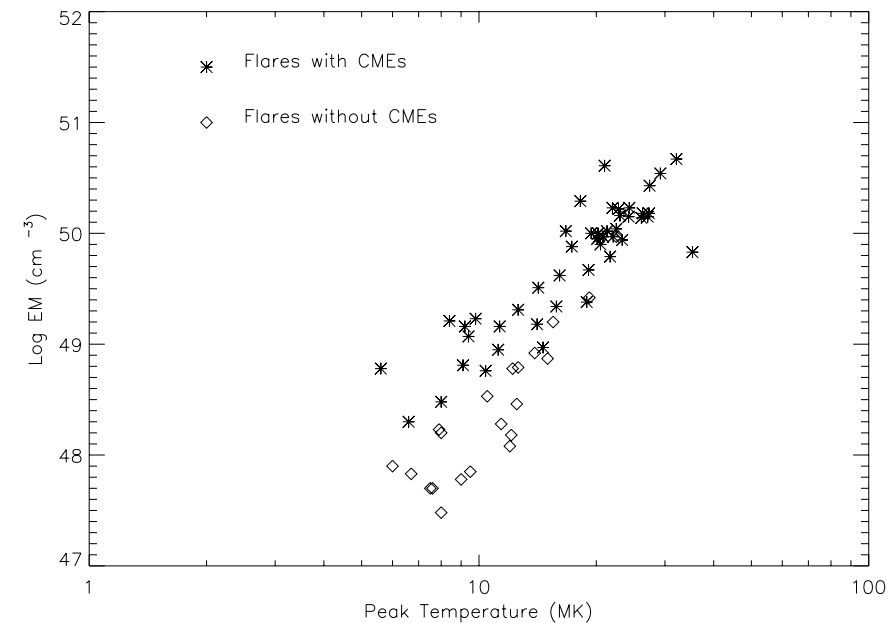

Fig. 3. The relationship between peak temperature and peak emission measure.

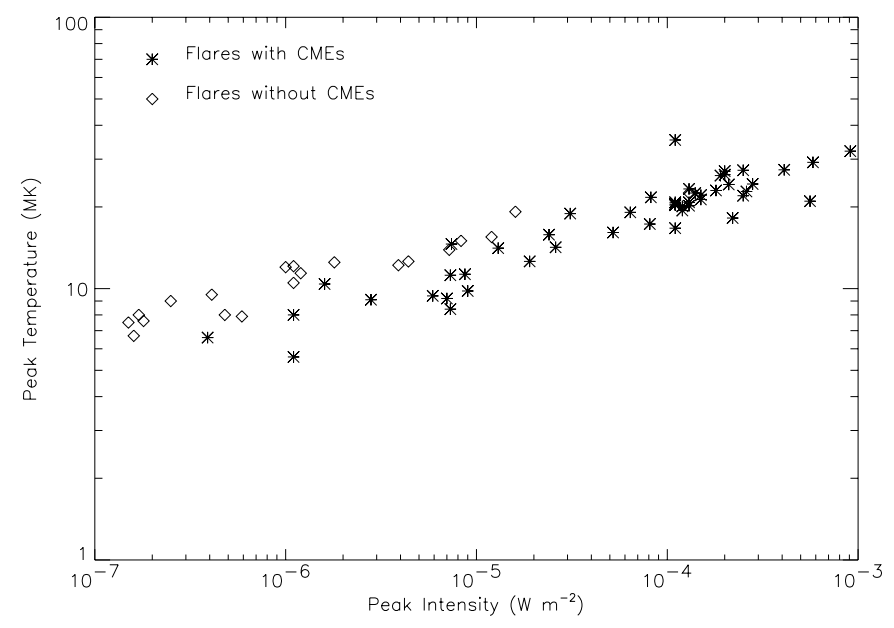

Fig. 4. The relationship between peak temperature and intensity.

\subsection{Flare rise and decay phases}

The relative times for the initial increase and subsequent decrease in intensity for the flares may provide further information on the heating and cooling processes. Rise and decay times for each flare were defined as the time from the start of the flare to the peak of the emission seen by GOES and the time from the peak to the flare end time respectively. A comparison between the relative length of the rise and decay phases of the sample of flares (Fig. 5) indicates a clear correlation with the length of rise phase increasing with increasing decay phase. However, there is no apparent difference in this relationship for ejective and non-ejective events. Considering the peak temperature of the events (Fig. 6) there is a tendency for events without associated CMEs to reach a given temperature faster than those which were associated with CMEs. This may be due to the confined nature of the events without associated CMEs.

\subsection{EIT waves}

There are many other transient solar events which occur in conjunction with flares and CMEs, such as radio bursts and waves. 
Table 1. Summary of the mean temperatures and flare durations for the sample of flares. The numbers in brackets refer to the median values.

\begin{tabular}{lccc}
\hline \hline SXR Property & Whole Sample & Flares with CMEs & Flares without CMEs \\
\hline Mean peak temperature (MK) & $16.4(15.8)$ & $18.7(20.2)$ & $11.4(11.4)$ \\
Mean flare duration (min) & $185.4(127.0)$ & $246.1(161.0)$ & $58.3(26.0)$ \\
Mean decay/rise times & 8.24 & 9.35 & 5.92 \\
\hline
\end{tabular}

Table 2. Mean flare duration and peak temperature for each GOES class.

\begin{tabular}{c|cccccc|cccc}
\hline \hline GOES & \multicolumn{7}{|c|}{ Flare Duration (min) } & \multicolumn{4}{c}{ Peak Temperature (MK) } \\
Class & With CMEs & median & $n$ & Without CMEs & median & $n$ & With CMEs & median & Without CMEs & median \\
\hline B & 142.0 & - & 1 & 24.1 & 21.0 & 9 & 6.6 & - & 7.8 & 7.9 \\
C & 193.3 & 186.0 & 11 & 62.9 & 33.0 & 9 & 9.7 & 9.4 & 12.5 & 12.2 \\
M & 195.9 & 162.0 & 9 & 142.5 & 142.5 & 2 & 16.6 & 16.1 & 17.4 & 17.4 \\
X & 279.1 & 158.0 & 27 & 156.0 & - & 1 & 23.2 & 22.5 & 22.5 & - \\
\hline
\end{tabular}

Table 3. Upper and lower limits of the soft X-ray flare characteristics.

\begin{tabular}{lcc}
\hline \hline SXR Property & Flares with CMEs & Flares without CMEs \\
\hline Minimum flare duration (min) & 32 & 3 \\
Maximum flare duration (min) & 1630 & 291 \\
Minimum peak temperature (MK) & 6.6 & 6.0 \\
Maximum peak temperature (MK) & 35.3 & 22.5 \\
\hline
\end{tabular}

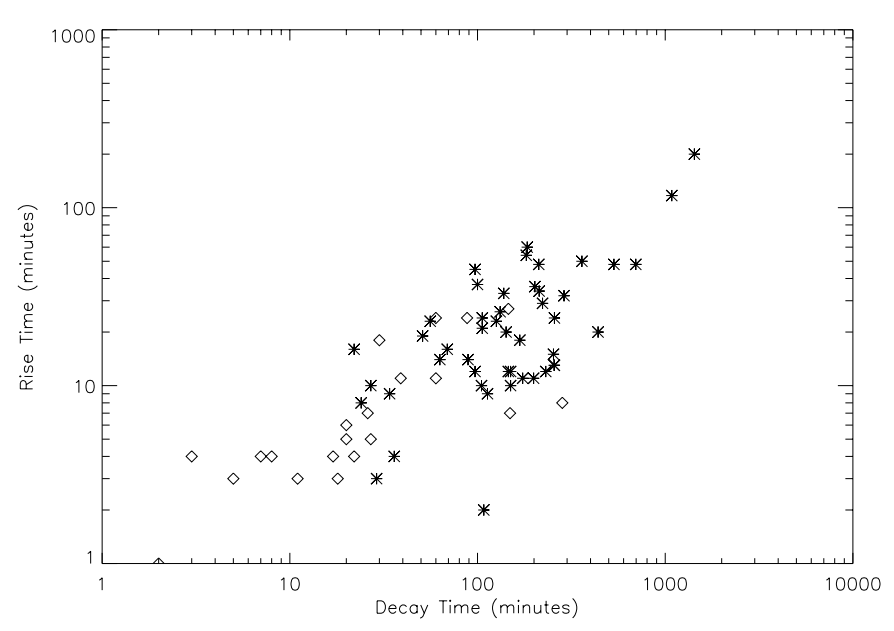

Fig. 5. The time from the start of the event to the time of peak intensity (rise time) as a function of the length of the decay phase for all of the events considered. While the rise time increases with increasing decay time, there do not appear to be any systematic differences resulting from the occurrence of a CME.

EIT waves are large scale propagating emission fronts, followed by expanding dimming regions, seen originating from the site of active region transients in EIT images (Moses et al. 1997). It has been suggested that these are the coronal counterparts of the chromospheric waves seen in $\mathrm{H} \alpha$ images, known as Moreton waves (Moreton 1960; Moreton \& Ramsey 1960), but with velocities of only one third that of the chromospheric waves (Klassen et al. 2000). Modelling done by Wang (2000) suggests that the basic properties of EIT waves including the avoidance of strong magnetic features, neutral lines

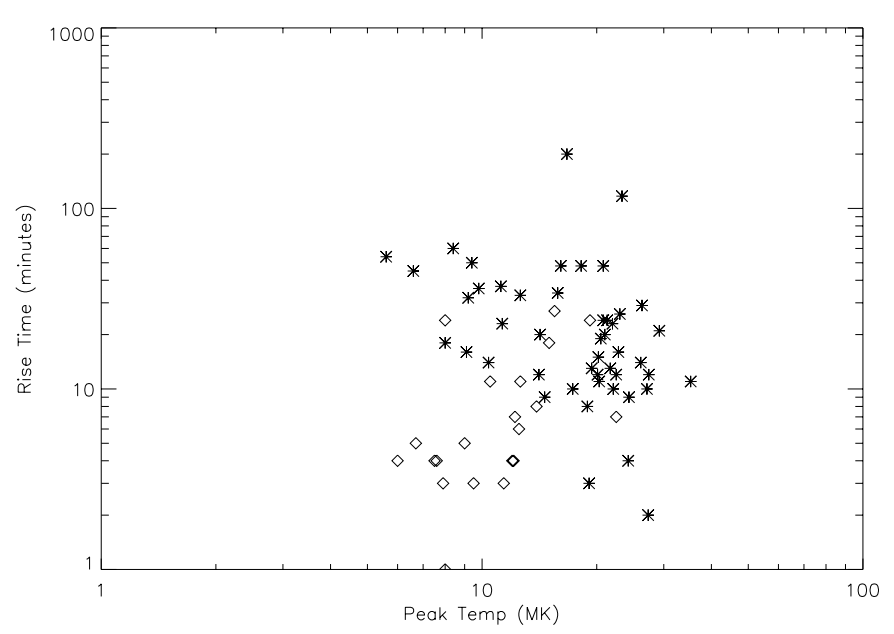

Fig. 6. Rise time of the flares as a function of the peak temperature, determined from a ratio of the GOES fluxes.

and coronal holes, can be accounted for by a fast-mode MHD hypothesis.

Chen et al. (2002) have performed numerical simulations of the formation of a CME and show that the piston-driven shock formed as a flux rope rises is responsible for the type II radio bursts often seen in association with CMEs and that two wavelike features are formed; a fast wave at the outermost part of the perturbed region, corresponding to one leg of the piston-driven shock wave and a slower wave lying between this and the flare loop footpoint. This slower wave front is the EIT "wave" and is in fact due to the propagation of disturbances formed by the opening of magnetic field lines above the flux rope. The velocities of EIT waves are therefore dependent on the fast-mode wave velocity and are not related to the velocity of the flux rope 
as it rises. Chen (2002) postulates that the piston-driven shock may sweep the lower atmosphere to produce a Moreton wave, with the predicted velocities of both types consistent with observations.

Recent work by Biesecker et al. (2002) has shown that there is a strong correlation between EIT waves and CMEs, but less correlation with flares and type II radio bursts. For a sample of 173 EIT waves they have observed, after accounting for observational bias, they find a CME associated with each one. This would seem to be consistent with the Chen et al. model where it is only the opening of field lines which is important in the production of an EIT wave and not the occurrence of a flare.

Where sufficient EIT data were available, EIT waves were looked for in the events considered here. In total 11 EIT waves were identified, all of which were associated with CMEs, which supports the correlation between EIT waves and CMEs found by Biesecker et al. (2002). While we do not see EIT waves for the remaining $33 \mathrm{CME}$ associated events for which we have EIT data, it is possible that we are not able to detect all EIT waves either as a result of the poor cadence of EIT $(\sim 12 \mathrm{~min})$ or due to them being too faint to detect with EIT. Of course it is also possible that there is some inherent property of these particular CME events which precludes the formation of an EIT wave. A comparison between the occurrence of an EIT wave and the speed of the CME (taken from the linear fits to LASCO CME time-height plots in the CME catalogue of Yashiro), has shown no correlation, with waves being associated with CMEs of speeds from $\sim 200-1800 \mathrm{~km} \mathrm{~s}^{-1}$. In addition, the waves do not seem to be preferentially associated with either high peak intensity flares, or flares with particularly extreme rise or decay times.

\section{Discussion}

The comparison between the X-ray properties of the flares has highlighted subtle differences between the flares with and without an associated CME. One difficulty in making comparisons between the two types of events is the interdependency of the observed parameters. For example, both the peak temperature and emission measure are coupled with the peak intensity reached in the event.

For a given peak intensity, flares where mass has been ejected appear to reach lower peak temperatures, up to mid-M class, than corresponding events which are confined. This suggests that when a CME occurs less energy goes into heating the plasma, with the remainder presumably going into the ejection of material in the CME. This is supported by the work of Green et al. (2001), who looked at the CME and flare history of nine active regions at solar minimum. Considering both the duration and intensity of the flares Green et al. (2001) suggest that for a given active region, CME activity removes energy from the region, resulting in less energy being available for flares. In the regions they studied the flares occurring after a CME from the region tended to be of lower intensity and shorter duration. They did not consider however the association of the CME with any particular flare and hence did not consider the effect of the $\mathrm{CME}$ on the heating occurring within the flare.
If we consider the results presented here in terms of a given peak temperature, then flares with associated CMEs have higher peak intensities. If, as suggested by Moore et al. (2001), reconnection stops abruptly when the explosion is confined in the case of non-eruptive events, but continues for much longer in the case of ejective events then perhaps we would expect to see higher peak intensities and longer durations for eruptive events. Additionally, for a given peak temperature we see lower values for the peak emission measure in the case of nonCME events, which when combined with the shorter rise times seen for these events seems compatible with the view that a shorter time is required to heat the plasma to the given peak temperature.

Eleven EIT waves were seen in the cases where there were sufficient EIT data, and all were associated with CMEs. However, the occurrence of waves was not associated with any of the soft X-ray parameters and in fact waves were seen for flares with a range of GOES classes and CME speeds. There did not appear to be evidence of any underlying reason as to why EIT waves were, or were not, associated with any particular event.

\section{Conclusions}

The relationship between flare duration, peak intensity, emission measure and temperature is investigated for a sample of solar flares, both with and without associated CMEs. A statistical comparison between the two classes of flares has highlighted the following relationships;

- There is a clear correlation between peak intensity and flare duration for the events without CMEs, with no correlation in the case of the flares which did have associated CMEs, suggesting that the occurrence of a CME intrinsically affects the timescale for energy release and cooling within the flare.

- The same correlation between peak temperature and peak emission measure as that found by Feldman et al. (1995a, b) is seen for all of the events considered. However there do appear to be systematic differences between the two classes of events with flares without CMEs tending on average to reach higher temperatures than corresponding CME associated event, as seen in Fig. 3.

- In general, increasing rise time is accompanied by an increasing length of the decay phase, but there is no distinction between the two types of event.

- There seems to be no relationship between the rise time of a flare and the peak temperature the flare reaches, suggesting that the peak temperature is just determined by the amount of energy involved in the flare, and not the rate at which it is released.

- We find that eleven of the events (which have sufficient EIT data), are associated with EIT waves, all of which occur in conjunction with CMEs. We see no correlation between the occurrence of a wave and CME speed, flare intensity or rise time.

It is worth noting however that this study has considered only simple events, based on the soft X-ray time profile of the 
flares. We have selected only a small fraction of the huge numbers of GOES-class events which have occurred on the Sun since the launch of $S O H O$, although it is not unreasonable to assume that this is a representative sample. In addition we have concentrated only on the soft X-ray properties of the flares.

To enable a more complete comparison of the two types of event and probe more deeply into the energetics and kinematic processes responsible for producing the relationships found here, we intend to study the hard X-ray properties and non-thermal velocities of a subset of these events, using data from the Hard X-ray Telescope (HXT) and Bragg Crystal Spectrometer (BCS) on the Yohkoh satellite. In addition, as the morphology of the active region responsible for the production of the flare has been shown to play an important role in determining whether mass will be ejected, we intend to make a more detailed analysis of the EIT images and, where possible, employ data taken with the TRACE satellite in an effort to explore the flare-CME relationship further.

Acknowledgements. HRMK acknowledges financial support from the PPARC and LKH thanks PPARC for the award of an Advanced Fellowship. This work used data obtained from the Solar UK Research Facility (SURF) at Mullard Space Science Laboratory. The $S O H O / L A S C O$ data used in this work are produced by a consortium of the Naval Research Laboratory (USA), Max-Planck-Institut fuer Aeronomie (Germany)), Laboratoire d'Astronomie (France), and the University of Birmingham (UK). SOHO is a project of international co-operation between ESA and NASA.

\section{References}

Aschwanden, M. J. 1999, Sol. Phys., 190, 233

Biesecker, D. A., Myers, D. C., Thompson, B. J., Hammer, D. M., \& Vourlidas, A. 2002, ApJ, 569, 1009

Brueckner, G. E., Howard, R. A., Koomen, M. J., et al. 1995, Sol. Phys., 162, 357

Chen, P. F., Wu, S. T., Shibata, K., \& Fang, C. 2002, ApJ, 572, L99
Delaboudinière, J.-P., Artzner, G. E., Brunaud, J., et al. 1995, Sol. Phys., 162, 291

Feldman, U., Doschek, G. A., Mariska, J. T., \& Brown, C. M. 1995a, ApJ, 450, 441

Feldman, U., Laming, J. M., \& Doschek, G. A. 1995b, ApJ, 451, L79

Feldman, U., Doschek, G. A., Behring, W. E., \& Phillips, K. J. H. 1996, ApJ, 460, 1034

Garcia, H. A. 1994, Sol. Phys., 154, 275

Green, L. M., Harra, L. K., Matthews, S. A., \& Culhane, J. L. 2001, Sol. Phys., 200, 189

Green, L. M., Matthews, S. A., van Driel-Gesztelyi, L., Harra, L. K., \& Culhane, J. L. 2002, Sol. Phys., 205, 325

Harra, L. K., \& Sterling, A. C. 2001, ApJ, 561, L215

Harrison, R. A. 1995, A\&A, 304, 585

Harrison, R. A., Sawyer, E. C., Carter, M. K., et al. 1995, Sol. Phys., 162,233

Hudson, H. S., \& Webb, D. F. 1997, in Geophysical Monographs 99, Coronal Mass Ejections, ed. N. Crooker, J. Joselyn, \& J. Feynman (Washington: AGU), 27

Khan, J. I., \& Hudson, H. S. 2000, Geophy. Res. Lett., 27, 1083

Klassen, A., Aurass, H., Mann, G., \& Thompson, B. J. 2000, A\&AS, 141,357

Moore, R. L., Sterling, A. C., Hudson, H. S., \& Lemen, J. R. 2001, ApJ, 552, 833

Moreton, G. E. 1960, AJ, 65, 494

Moreton, G. E., \& Ramsey, H. E. 1960, PASP, 72, 357

Moses, D., Clette, F., Delaboudiniere, J.-P., et al. 1997, Sol. Phys., 175,571

Sheeley, N. R. Jr., Howard, R. A., Koomen, M. J., \& Michels, J. 1983, ApJ, 272, 349

Sterling, A. C., \& Hudson, H. S. 1997, ApJ, 491L, 55

Veronig, A., Temmer, M., Hanslmeier, A., Otruba, W., \& Messerotti, M. 2002, A\&A, 382, 1070

Wang, Y.-M. 2000, ApJ, 543, L89

Zarro, D. M., Sterling, A. C., Thompson, B. J., Hudson, H., \& Nitta, N. 1999, ApJ, 520, L139

Zhang, J., Dere, K. P., Howard, R. A., Kundu, M. R., \& White, S. M. 2001, ApJ, 559, 452 